Research Paper

\title{
Disialyl GD2 ganglioside suppresses ICAM-1-mediated invasiveness in human breast cancer MDA-MB231 cells
}

\author{
Kyung-Min Kwon ${ }^{1}, 2$, Tae-Wook Chung ${ }^{3 凶}$, Choong-Hwan Kwak ${ }^{1}$, Hee-Jung Choi ${ }^{3}$, Kyung-Woon Kim ${ }^{4}$, \\ Sun-Hyung Ha${ }^{1}$, Seung-Hak Cho ${ }^{5}$, Young-Choon Lee ${ }^{6}$, Ki-Tae Ha ${ }^{3}$, Moon-Jo Lee and Cheorl-Ho Kim ${ }^{1}{ }^{8} \bowtie$ \\ 1. Molecular and Cellular Glycobiology Unit, Department of Biological Sciences, Sungkyunkwan University, Seoburo 2066, Jangan-Gu, Suwon, Gyunggi-Do, \\ 16419, Korea; \\ 2. Research Institute, Davinch-K Co., Ltd., B1603-3, 606, Seobusaet-gil, Geumcheon-gu, Seoul 153-719, Korea; \\ 3. Division of Applied Medicine, School of Korean Medicine, Pusan National University, Yangsan City, Gyeongsangnam-Do, Korea; \\ 4. Animal Biotechnology Division, National Institute of Animal Science, RDA, Wanju-gun, Jeollabuk-do, 55365, Korea; \\ 5. Division of Enteric Diseases, Center for Infectious Diseases Research, Korea National Institute of Health, Heungdeok-gu, Cheongju 363-951, Korea; \\ 6. Department of Biotechnology, Dong-A University, Busan 604-714, Korea; \\ 7. Department of Herb Science, Dong-Eui Institute of Technology, 54, Yangji-ro, Busanjin-Gu, Busan 47230, Korea; \\ 8. Department of Medical Device Management and Research, Samsung Advanced Institute of Health Science and Technology (SAIHST), Seoul 06351, Korea. \\ $\triangle$ Corresponding authors: Cheorl-Ho Kim chkimbio@skku.edu Fax: +82-31-290-7015; Tae-Wook Chung twchung@pusan.ac.kr \\ (C) Ivyspring International Publisher. This is an open access article distributed under the terms of the Creative Commons Attribution (CC BY-NC) license \\ (https://creativecommons.org/licenses/by-nc/4.0/). See http://ivyspring.com/terms for full terms and conditions.
}

Received: 2016.07.20; Accepted: 2016.12.12; Published: 2017.02.12

\begin{abstract}
The disialoganglioside GD3 has been considered to be involved in tumor progression or suppression in various tumor cells. However, the significance of the biological functions of GD3 in breast cancer cells is still controversial. This prompted us to study the possible relationship(s) between GD3 expression and the metastatic potential of a breast cancer MDA-MB231 cells as an estrogen receptor negative (ER-) type. The human GD3 synthase cDNA was transfected into MDA-MB231 cells, and G-418 bulk selection was used to select cells stably overexpressing the GD3 synthase. In vitro invasion potentials of the GD3 synthase over-expressing cells (pc3-GD3s) were significantly suppressed when compared with control cells. Expression of intercellular adhesion molecule-1 (ICAM-1; CD54) was down-regulated in the pc3-GD3s cells and the decrease in ICAM-I expression is directly related to the decrease in invasiveness of the pc3-GD3s cells. Another type of ER negative SK-BR3 cells exhibited the similar level of ICAM-1 expression as MDA-MB231 cells, while the ER positive MCF-7 cells $(E R+)$ showed the increased expression level of ICAM-1. Then, we investigated signaling pathways known to control ICAM-1 expression. No difference was observed in the phosphorylation of ERK and p38 between the pc3-GD3s and control cells (pc3), but the activation of AKT was inhibited in pc3-GD3s, and not in the control (pc3). In addition, the composition of total gangliosides was changed between control (pc3) and pc3-GD3s cells, as confirmed by HPTLC. The pc3-GD3s cells had an accumulation of the GD2 instead of the GD3. RT-PCR results showed that not only GD3 synthase, but also GM2/GD2 synthase ( $\beta 4-G a l N c$ T) expression was increased in pc3-GD3s cells. Overexpression of GD3 synthase suppresses the invasive potential of human breast cancer MDA-MB-231 cells through down-regulation of ICAM-1 and the crucial pathway to allow the apoptotic effect has been attributed to accumulation of the GD2 ganglioside. ER has been linked to the ICAM-1 expression with GD3 to GD2 conversion in human breast cancer cells. This is the first finding of the endogenous sialyltransferase functions in tumor cells.
\end{abstract}

Key words: Ganglioside GD3 synthase, Breast cancer, MDA-MB231, Intracellular adhesion molecule-1, GM2/GD2 synthase ( $\beta 4-G a l N c T)$, Invasion.

\section{Introduction}

Gangliosides are sialylated glycosphingolipids which are ubiquitously expressed in mammalian plasma membranes and they play crucial roles in cellular interaction, adhesion, differentiation and apoptosis [1, 2]. To synthesize gangliosides in cells, lactosyl ceramide (Lac-Cer) is processed by different 
pathways composed of various glycosyltransferases or sialyltranferases $[1,3]$. One of these sialyltransferases, GD3 synthase (ST8Sia I), is a key enzyme which controls GD3 biosynthesis from its precursor ganglioside GM3 [3; 4]. The steady state level of GD3 expression also depends on other enzymes such as GD2 synthase and GT3 synthase. Thus, the relationship between a ganglioside and its related enzymes is linked to its glycosylation pattern in the cell membrane. The GD3 is expressed weakly in normal tissues [5], and expressed almost exclusively during development or under pathological conditions such as neuronal disorders [6]. In addition, GD3 promotes tumor progression by influencing cellular proliferation, adhesion and metastasis in malignant tumors $[7,8]$. However, GD3 is also known to induce apoptosis [5] by transiently accumulating in the cytosol to contribute to mitochondria damage in the early stages of apoptosis [5, 9]. Fas-mediated apoptosis was also induced by GD3 in lymphoid cells [10]. Therefore, the significance of the biological functions of the ganglioside GD3 in cancer cells is still controversial.

GD3 has long been reported to be highly expressed in breast tumors, the most common malignant women's disease, although at a lower level than the ganglioside GM3 [11]. The GD3 synthase was also reported to be associated with breast tumor [12]. Although the properties of breast cancers and breast cancer cell lines are quite different, extensive studies on the relationship between GD3 expression and the metastatic potential of human breast cancer cells have not been reported. The GD3 synthase expression is also linked to the activation of the c-Met signaling pathway for the metastatic potentials [13]. In a recent report [14], it was further demonstrated that the GD3 synthase expression is regulated by several transcription factors which are related to the epithelial-mesenchymal transition (EMT) pathway. The EMT-mediated cancer cells exhibit the metastatic potential in the breast cancer cells. For the disialoganglioside GD2, it was previously known that the cancer stem cells express the GD2 in the breast cancer cells, where GD2 synthesis is dependent on the GD3 synthesis [15]. GD2 is synthesized from GD3 by the GD2 synthase and GD3 synthase activity is prerequisite for GD2 synthesis. Therefore, GD2 has been suggested as a breast cancer stem cell marker. The expression of GD3 synthase correlates with poor prognosis in human breast tumors. Ganglioside GD2 as a b-series ganglioside has been reported to be expressed on bone marrow-derived mesenchymal stem/stromal cells [16]. It has also been reported that antibodies against GD2 inhibits proliferative phenotype of tumor cells, triggering apoptotic cell death in small cell lung cancer cell lines (SCLC) [11]. Recently, our group reported that the apoptotic functions of disialyl GD1b in human breast cancer MCF-7 cells [2], where endogenous and exogenous GD1b effectively promoted apoptotic cell death in human breast carcinoma SKBR3 cells.

Intercellular adhesion molecule-1 (ICAM-1) is an immunoglobulin superfamily glycoprotein located on the cell surface. It is well known that ICAM-1 is associated with various inflammatory systems and immune responses $[17,18]$. It was also reported that the elevated ICAM-1 expression positively correlates with the metastatic and invasive potential of pancreatic, gastric and breast cancer cells [19, 20, 21]. In addition, inhibition of ICAM-1 function by siRNA treatment resulted in a strongly decreased invasive potential of breast cancer cell [20]. Recently, Schröder et al. (2011) reported that the ICAM-1 expression was associated with more aggressive tumor phenotype and prognosis in the breast cancer patients [22].

The human breast cancer cell line, MDA-MB231, is a relative highly metastatic and invasive breast cancer model [23, 24]. Although these cells express higher levels of ICAM-1 than those of other breast cancer cell lines [20], expression levels of GD3 and GD3 synthase are very low. In this study, we have for the first time studied on the relation of ganglioside GD3 and ICAM-1 in breast cancer MDA-MB231 cells. The expression levels of GD3 synthase (ST8Sia I) were compared in different breast cancer cell lines for the effect of overexpression of GD3 synthase on invasive potential. Interestingly, the overexpressed GD3 has been further converted to the GD2 ganglioside by the endogenous GD2 synthase activity. This is a first finding of GD2 synthetic pathway during GD3 synthesis. Thus, the relationship between GD3 synthase (ST8Sia I) and ICAM-1 expression has been discussed in MDA-MB231 cells with the crucial factor of GD2 biosynthesis.

\section{Materials and methods}

\section{GD3 expression plasmid and transfected cells}

The human breast cancer cell lines of ER positive MCF-7 (ER+), ER negative SK-BR3 (ER-) and ER negative MDA-MB231 (ER-) cells [2, 23] were cultured in Dulbecco's modified Eagle's medium (DMEM) supplemented with $10 \%$ or $5 \%$ fetal bovine serum respectively. To construct the GD3 synthase expressing plasmid, a 1026 bp DNA fragment including the human GD3 synthase coding region was amplified by PCR using the primers sense (5'-GATATCGCCGCCACCATGGCTGTACTGGCGT GGAAG-3') and antisense (5'-CTCGAGCTAGGAAG TGGGCTGGAGTGA-3'), and human melanoma 
cDNA as template. The PCR fragment was ligated into pcDNA3 vector (pc3), to generate pcDNA3-GD3 synthase (pc3-GD3s). The pc3-GD3s was transfected into the MDA-MB231. The transfected cells were plated in 10-cm culture dish for $48 \mathrm{~h}$ after transfection. Isolation of the stable trasfectants were accomplished by adding Geneticin (G418 sulfate; Gibco BRL) to the culture medium at $1 \mathrm{mg} / \mathrm{ml}$. G418-resistant transfected clones appeared in about 2 weeks, and more than clones were pooled later on and expanded.

\section{Reverse transcription PCR and real time quantitative PCR}

Total RNA was extracted from cultured cells and cDNA was prepared using first-strand cDNA synthesis kit for reverse transcription-polymerase chain reaction (RT-PCR) (M-MLV RT) (RexGene biotech, Korea). cDNAs were amplified by PCR with primer set I (Table 1$)$. The housekeeping gene, $\beta$-actin, was used as an endogenous control to normalize RNA extractions. PCR reactions $(20 \mu \mathrm{l})$ were performed using 2x Super Taq master PCR premix (RexGene biotech, Korea). For real-time quantitative PCR, the cDNA was amplified with primer set II (Table 2). Real-time quantitative PCR assays were controlled by analyzing the expression levels of the housekeeping gene GADPH. Real-time quantitative PCR was performed using the SYBR green system (Bio-RAD, USA). The real-time reactions $(20 \mu \mathrm{l})$ were performed with $\mathrm{iQ}^{\mathrm{TM}} \mathrm{SYBR}{ }^{\mathrm{B}}$ Green Supermix reagent (Bio-RAD, USA), and analyzed by Opticon Monitor 3 (Bio-RAD, USA).

Table 1. RT-PCR primer sequences

\begin{tabular}{|c|c|c|c|}
\hline Genes & Primers & $\begin{array}{l}\text { Anneling } \\
\text { Temp. }\left({ }^{\circ} \mathrm{C}\right)\end{array}$ & $\begin{array}{l}\text { PCR } \\
\text { product } \\
\text { size (bp) }\end{array}$ \\
\hline GD3 synthase & $\begin{array}{l}\text { Fwd: atggctgtactggcgtggaagt } \\
\text { Rev: atgtctttctggaccacagaaggt }\end{array}$ & $55^{\circ} \mathrm{C}$ & $571 \mathrm{bp}$ \\
\hline GM3 synthase & $\begin{array}{l}\text { Fwd: atccagttatcatcaaagagactgc } \\
\text { Rev: atacatcaagaaggctgtcaaaaac }\end{array}$ & $55^{\circ} \mathrm{C}$ & $373 \mathrm{bp}$ \\
\hline ICAM-1 & $\begin{array}{l}\text { Fwd: cagtgaccatctacagctttccgg } \\
\text { Rev: gctgctaccacagtgatgatgacaa }\end{array}$ & $55^{\circ} \mathrm{C}$ & $556 \mathrm{bp}$ \\
\hline GM2/GD2 synthase & $\begin{array}{l}\text { Fwd:atcggctacgggctctcatcacc } \\
\text { Rev:acctcgcgcaccttgtcagtcc }\end{array}$ & $55^{\circ} \mathrm{C}$ & $478 \mathrm{bp}$ \\
\hline$\beta$-actin & $\begin{array}{l}\text { Fwd: caagagatggccacggctgct } \\
\text { Rev: tccttctgcatcctgtcggca }\end{array}$ & $55^{\circ} \mathrm{C}$ & $247 \mathrm{bp}$ \\
\hline
\end{tabular}

Table 2. Real-time PCR primer sequences

\begin{tabular}{|c|c|c|c|}
\hline Genes & Primers & Anneling Temp. $\left({ }^{\circ} \mathrm{C}\right)$ & Ref. \\
\hline GD3 synthase & $\begin{array}{l}\text { Fwd: gcgatgcaatctccetcct } \\
\text { Rev: ttgccgaattatgctgggat }\end{array}$ & $62^{\circ} \mathrm{C}$ & 4 \\
\hline ICAM-1 & $\begin{array}{l}\text { Fwd: cctgatgggcagtcaacagcta } \\
\text { Rev: acagctggctcccgtttca }\end{array}$ & $62^{\circ} \mathrm{C}$ & 32 \\
\hline GAPDH & $\begin{array}{l}\text { Fwd: aggtcatccctgagctgaacgg } \\
\text { Rev: cgcctgcttcaccaccttcttg }\end{array}$ & $62^{\circ} \mathrm{C}$ & 44 \\
\hline
\end{tabular}

\section{XTT proliferation assay and Western blot analysis}

Cell proliferation has been assayed using XTT assay solution (Sodium 3'-[1-(phenyl-aminocarbonyl)-3, 4-tetrazolium]-bis(4-methoxy-6-nitro) benzene sulfonic acid hydrate) [21]. To detect target proteins, we incubated the membranes with antibodies against ICAM-1(sc-7891), AKT(sc-1618), p-AKT(sc-135650), ERK(sc-154), p-ERK(sc-7383), p38(sc-535) and p-p38(sc-7973), were purchased from Santa Cruz (USA). Glyceraldehydes-3-phosphodehydrogenase (GAPDH) (MAB-8145) (Chemicon, USA), SARK/JNK(\#9252), p-SARK/JNK(\#9255) (Cell signaling, USA) and GD3 synthase (xw-8145) (ProSci incorporated, USA) were purchased. Detection was performed using a secondary antibodies and the ECL chemiluminescence system (RPN3000) (Amersham, UK).

\section{Invasion assay and flow cytometry}

Cell invasive potentials have been assessed using the gelatin-coated invasion chambers, as described [21]. For flow cytometry, cell surface expression of ICAM-1 (CD54) was analyzed by FACScan (Becton Dickinson, USA) using mouse anti-human ICAM-1 (CD54). The cells were incubated with mouse ICAM-1 antibodies (Santa Cruz, USA) and stained with FITC-conjugated goat anti-mouse IgG (Santa Cruz, USA) for $1 \mathrm{~h}$. Control cells were prepared using the second antibody alone.

\section{Reporter gene constructs and luciferase assays}

The ICAM-1 promoter regions spanning -1350 to +45 bp (full length) and -485 to +45 (truncated form) of the human ICAM-1 promoter were cloned into the vector pGL3-basic (Promega, USA). MDA-MB231 cells were transfected with $1 \mu \mathrm{g}$ of the plasmids or the control pCMV-Renilla plasmid using Wel-Fect EXTM Plus reagents (Wel-Gene, Korea). Cell extracts were prepared $24 \mathrm{~h}$ after transfection, and luciferase assays were performed using the Dual-Luciferase Reporter Assay System (Promega, USA). Luciferase activities were normalized with respect to parallel Renilla activities, to correct for differences in transfection efficiency.

\section{High-performance thin-layer chromatography (HPTLC) of gangliosides}

Ganglioside isolation has been described previously [26]. The ganglioiside fraction was eluted with chloroform: methanol : $0.8 \mathrm{M}$ sodium acetate (30: 60: 8, by vol.; Solvent B) and desalted using a Sep-Pac C18 cartridge (Millipore, USA). HPTLC analysis of gangliosides was performed using HPTLC silica gel plates (No. 113748; Merck, Germany, size $10 \times 10$ cm; 
thickness of the silica gel, $0.2 \mathrm{~mm}$ ) as described [27]. The TLC plate was stained using $0.2 \%(\mathrm{w} / \mathrm{v})$ orcinol in $20 \% \mathrm{H}_{2} \mathrm{SO}_{4}$.

\section{Results}

GD3 synthase gene expression patterns in human breast cancer cells

Three different breast cancer cell lines of MDA-MB231, MCF7 and SK-BR3 have been used for the GM3 synthase and GD3 synthase gene expression. MDA-MB231 cells show an invasive phenotype and are hormone-independent [28], and are an aggressive breast cancer model compared to MCF7 or SK-BR3 cells. As shown in Fig. 1A, the breast cancer cell lines expressed GM3 synthase mRNA. However, GD3 synthase mRNA was scarcely detected, while a melanoma SK-Mel II expressed largely the GD3 synthase.

\section{(A}

MCF-7 SK-Br3 MDA-MB231 SK-mel II (low invive) (lowinvsive) (highinvasive) (melanoma)

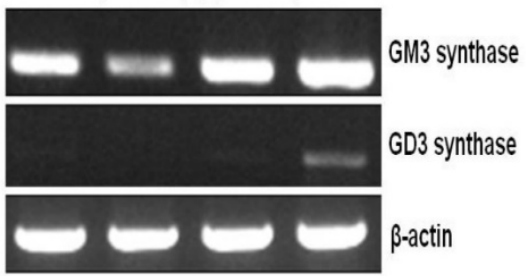

(28cycles)

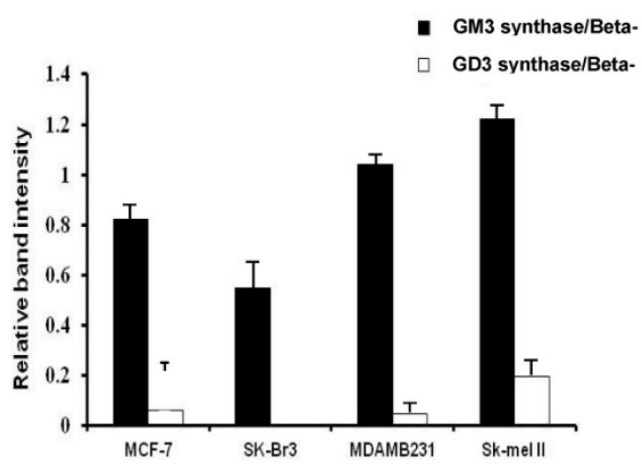

(B

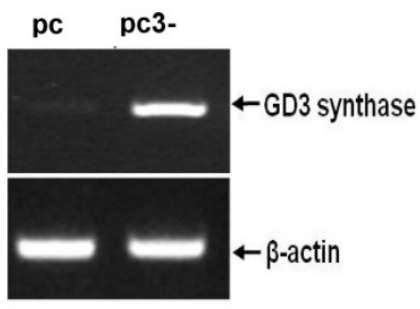

(C

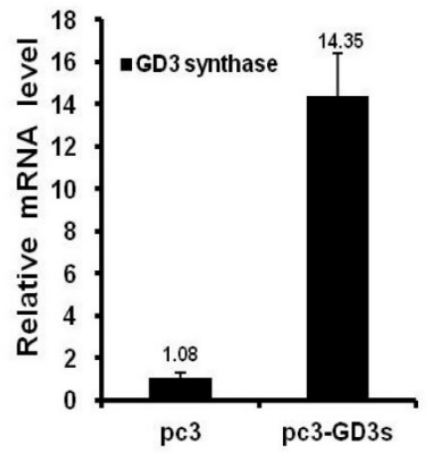

(D
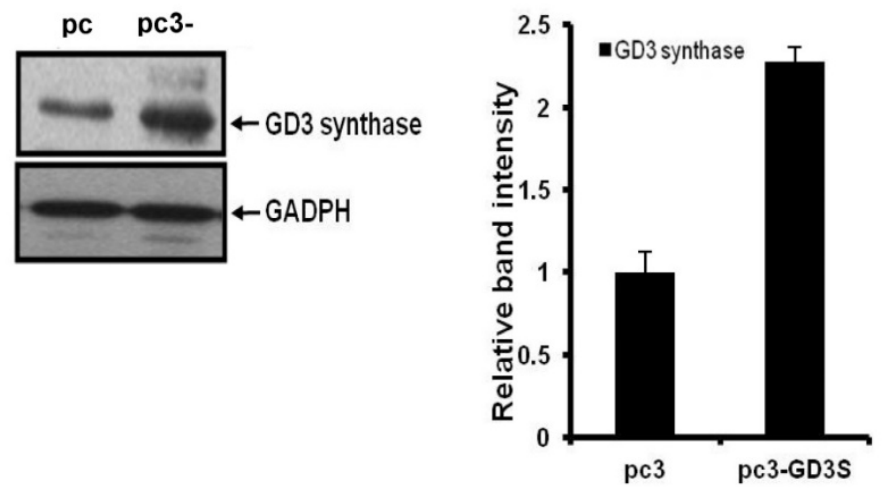

Figure 1. A) GD3 synthase gene expression patterns in human breast cancer cells. Total mRNA was isolated from breast cancer and SK-Mel II melanoma cells. The bar graphs represent the relative band intensity obtained from RT-PCR results by densitometry. For the expression levels of GD3 synthase mRNA, RT-PCR (B) and real-time PCR (C) analyses were performed. The protein expression was analyzed by Western blot (D). The pc3-GD3s cells were cultured with $1 \mathrm{mg} / \mathrm{ml}$ G-418. All experiments have been performed at least three times and we examined mean differences between groups by using the error bar graph procedure. 


\section{Over-expression of GD3 synthase in MDA-MB231 cells}

MDA-MB231 cells were transfected with the human GD3 synthase (pc3-GD3s) or the pcDNA3 empty vector as a control (pc3) (Fig. 1). RT-PCR and qPCR analysis clearly show that the induction of GD3 synthase mRNA occurred in the pc3-GD3s cells. There was a 14-fold induction of GD3 synthase mRNA level in pc3-GD3s than in pc3 cells (Fig. 1B and 1C). Elevated level of GD3 synthase protein was also observed in the pc3-GD3s cells (Fig. 1D). These results indicate that the pc3-GD3s cells overexpress the GD3 synthase mRNA and protein.

\section{GD3 synthase overexpressing cells show changes in morphology but not in cell growth}

We investigated whether GD3 synthase

\section{(A)}


(B)
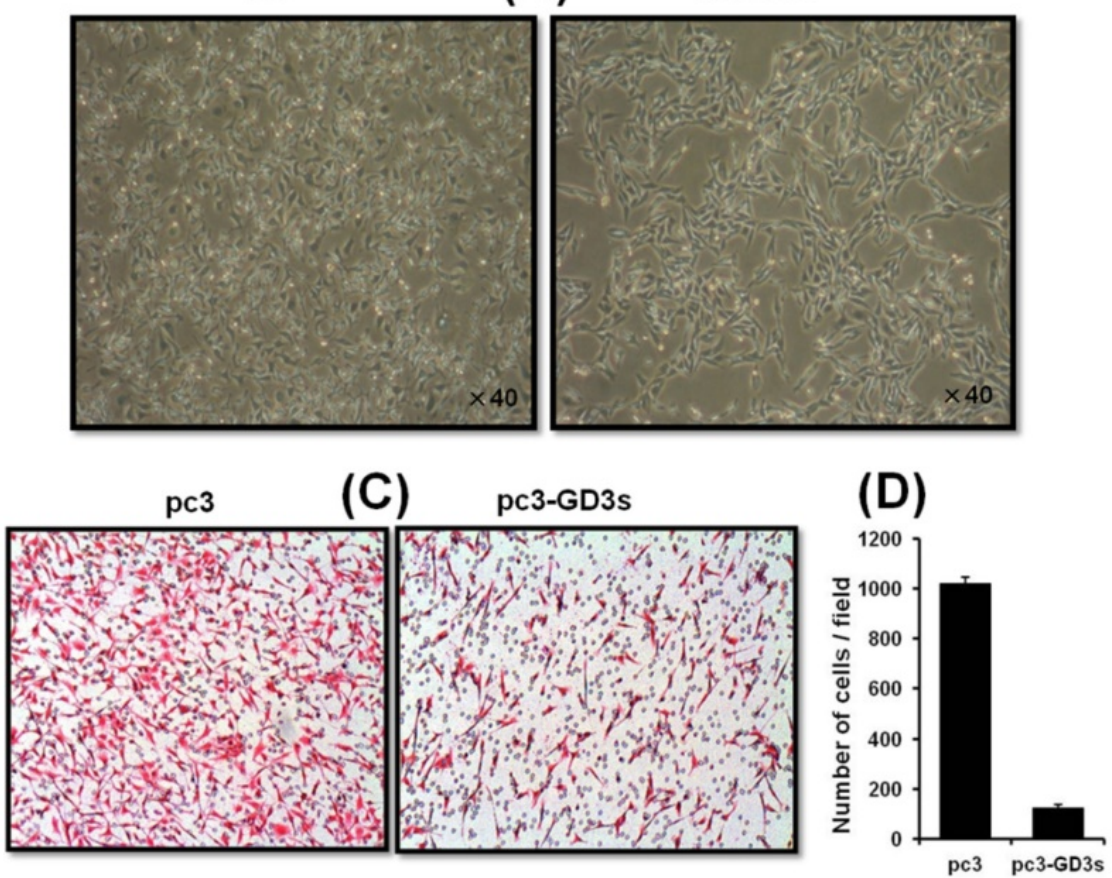

Figure 2. The pc3-GD3s cells show changes in morphology but not in cell growth Cell were cultured in DMEM supplemented with $10 \%$ or $5 \%$ FBS. The pc3 $(\bullet)$ and pc3-GD3s cells $(\boldsymbol{\square})$ were plated in triplicates. Cell growth in different serum conditions was assessed by the XTT assay after $24 \mathrm{hr}(\mathrm{A})$. The cell morphology was observed by phase contrast microscopy (B). Inhibition of invasiveness in GD3 synthase-transfected MDA-MB231 cells (C). Representative microphotographs of Hematoxylin and Eosin stained cells. Cells that invaded through the gelatin and were located on the underside of the filter were counted. All experiments performed at least three times and we examined mean differences between groups by using the error bar graph procedure. overexpression affects the cell growth of breast cancer cells. As shown in Fig. 2A, there was no difference between the cell growth of pc3-GD3s cells and pc3 cells when cultured with DMEM-10\% FBS or DMEM-5\% FBS. Although it was examined whether the suppressed ICAM-1 levels affect cell growth level of MDA-MB231 cells in vitro, no change in growth level was observed even by the specific ICAM1-1 siRNA (data not shown). More specifically, MDA-MB231 cells transfected with ICAM-1 siRNA exhibited the normal growth pattern as controls. Similar results were also obtained for MCF-7 breast cells, indicating that ICAM-1 is not related to the cell growth. However, significant morphological changes were observed in the pc3-GD3s cells compared with the pc3 cells. The arm-like projections observed in pc3 cells disappeared in pc3-GD3s cells, reducing the distance between the cells (Fig. 2B). The cell surface was observed to be smoother, suggesting that over-expression of GD3 synthase induces phenotype changes in the pc3-GD3s cells.

\section{Inhibition of the invasiveness of the GD3 synthase-transfected breast cancer cells}

To investigate the cell motility of pc3-GD3s cells, a transwell invasion assay was used. Invasion activity of the pc3-GD3s cells was suppressed 10-fold compared to the pc3 cells (Fig. 2C). This result indicates that overexpression of GD3 synthase in MDA-MB231 cells leads to changes in metastatic potential. Therefore, we hypothesized that the GD3 ganglioside regulates expression of invasion-related molecules in GD3 synthase-overexpressing cells.

\section{Downregulation of intracellular adhesion molecule 1 (ICAM-1) expression in pc3-GD3s cells}

The expression of ICAM-1 protein positively correlates with the metastatic potential of various human cancer cells [20, 28]. The expression of ICAM-1 was significantly suppressed in the MDA-MB231 cells that over-expressed GD3 synthase, as 
confirmed by RT-PCR and qPCR (Fig. 3A and 3B). In addition, protein expression of ICAM-1 was also suppressed by Western blot analysis and FACS analysis for the cell surface-localized form (Fig. 3C and 3D). We also utilized the cloned ICAM-1 promoter to assess transcriptional activity [29]. The full-length $1.4 \mathrm{~kb}$ ICAM-1 promoter contains several transcription factor binding sites, including ARE, Ap1 and TATA sites, while the truncated $0.5 \mathrm{~kb}$ promoter contains one each of NF-KB, STAT-1, TATA and AP1 sites. Each ICAM-1 luciferase reporter construct was cotransfected with the GD3 synthase gene into MDA-MB231 cells, or transfected without GD3 synthase. $24 \mathrm{~h}$ after co-transfection, the transcriptional activity of both the full-length and truncated forms of ICAM-1 promoter was two-fold downregulated by GD3 synthase cotransfection (Fig. 3E). These results suggest that the over-expression of GD3 synthase downregulates the ICAM-1 promoter activity in breast cancer cells.
(A)

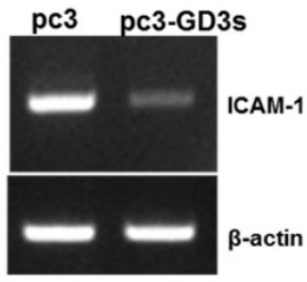

(B)

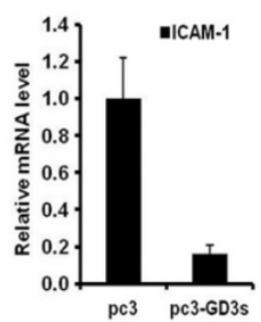

(C)

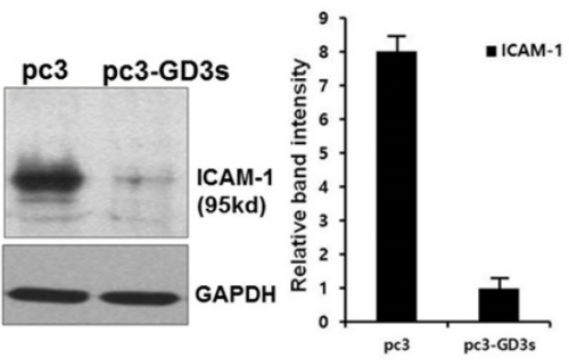

(D)

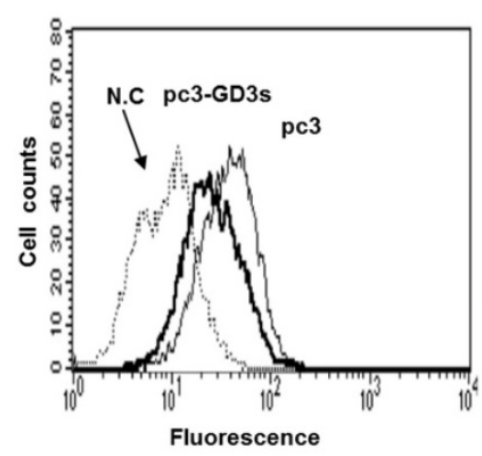

(E)

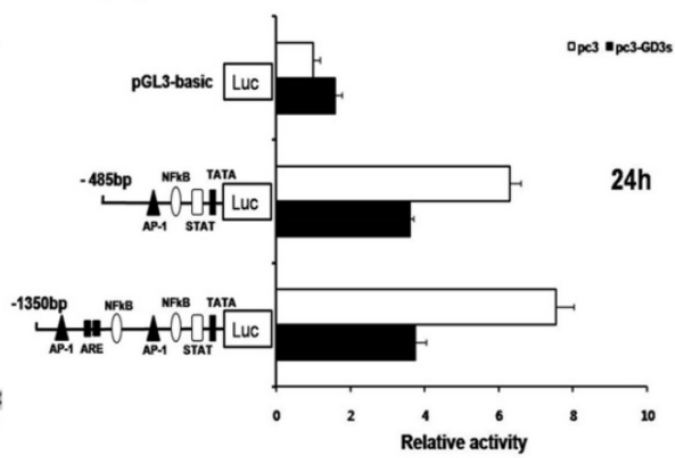

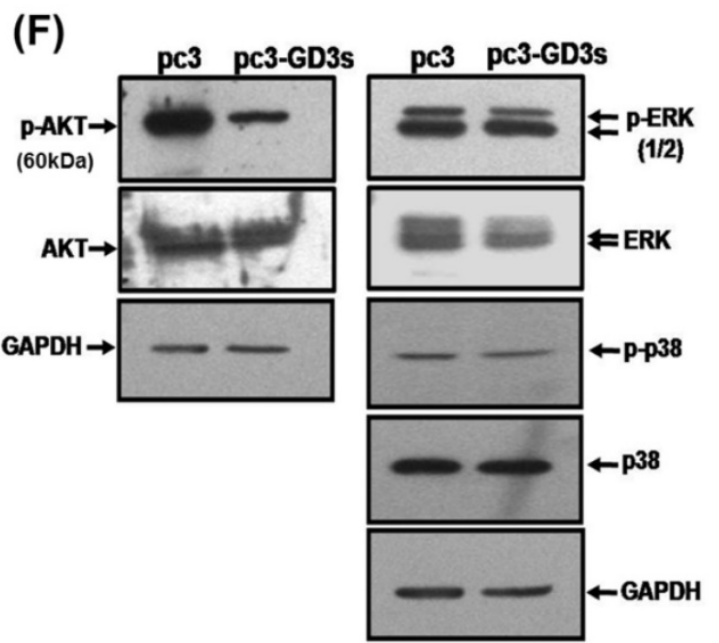

Figure 3. Downregulation of ICAM-1 expression (A, B, C, D, E) in pc3-GD3s cells. Expression levels of ICAM-1 mRNA were checked by RT-PCR (A) and real-time PCR (B). Expression of ICAM-1 confirmed by Western blot (C) and FACS analysis (D). A schematic representation of DNA constructions containing two different forms of the ICAM-1 promters linked to the luciferase reporter gene is shown. The values represent the mean \pm S.D. for three independent experiments with triplicate measurements (E). Inhibition of AKT phosphorylation in pc3-GD3s cells (F). Total protein extracts from pc3 and pc3-GD3s cells were examined by Western blot analysis with anti-phospho-AKT, anti-AKT, anti-phospho-ERK1/2, anti-ERK1/2, anti-phospho-p38 and anti-p38 antibodies. The nonspecific IgG antiserum has been used to block the cell reaction. GAPDH was included as an internal control. All experiments performed at least three times and we examined mean differences between groups by using the error bar graph procedure. 
(A)

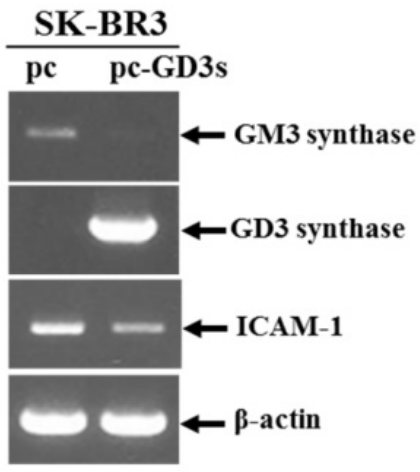

(C)

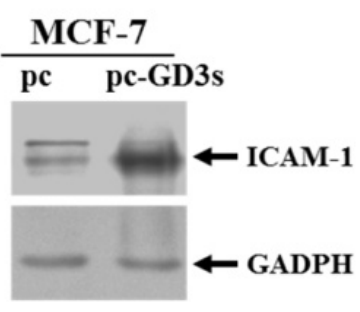

(B)

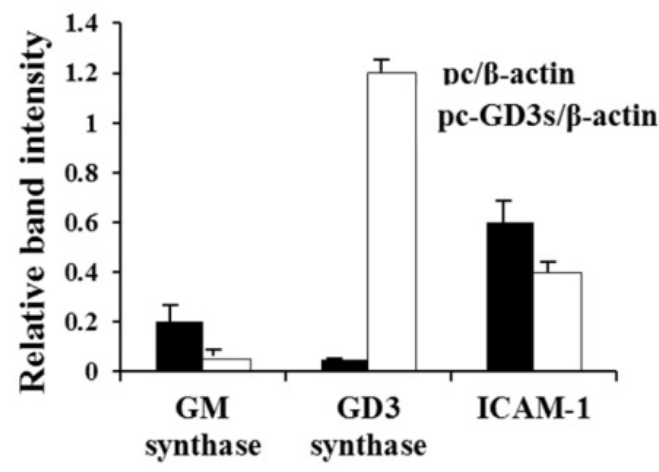

(D)

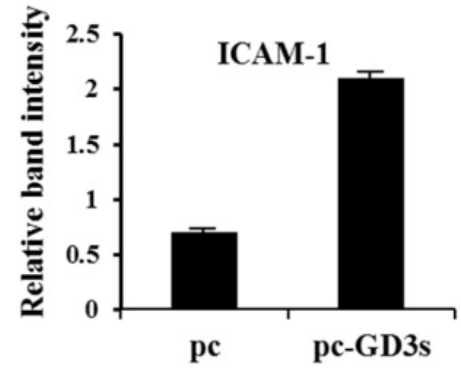

Figure 4. Differential regulation of ICAM-1 expression in pc3-GD3s-trasnfected SK-BR3 (ER-) and MCF-7 (ER+) cells. Expression levels of GM3 synthase, GD3 synthase and ICAM-1 mRNAs in the pc3-GD3s-transfected SK-BR3 (ER-) were checked by RT-PCR (A) and relative band intensities were compared with each $\beta$-actin control (B). $\beta$-Actin was included as an internal control. For MCF-7 (ER+) cells, ICAM-1 mRNA level in the pc3-GD3s-transfected MCF-7 cells was examined by Western blot analysis (C) and relative band intensity was also compared with GAPDH control (D). All experiments performed at least three times. We examined mean differences between groups by using the error bar graph procedure.

\section{Suppression of AKT phosphorylation in pc3-GD3s cells}

To better understand the pathways inactivated when ICAM-1 is suppressed, we investigated signaling-related phosphorylation using protein extracts from pc3 and pc3-GD3s cells. Western blot analysis on pc3-GD3s cells significantly showed down-regulation of AKT phosphorylation under 10\% serum conditions (Fig. 3F). However, other molecules such as p-ERK or p38, which are known to have roles in ICAM-1 expression, did not show a change in phosphorylation levels between the pc3 and pc3-GD3s cells. These results suggest that ICAM-1 expression is down-regulated in the pc3-GD3s cells through the inhibition of AKT phosphorylation.

\section{Decreased expression of ICAM-1 in ER-negative breast cancer SK-BR3 (ER-) and ER-positive MCF-7 (ER+) cells when transfected with pc3-GD3s}

In order to observe the similar phenomenon in another type of human breast cancer cells, estrogen receptor (ER) negative breast cancer cell line, SK-BR3 (ER-) has been examined whether the cells express the ICAM-1 mRNA upon transfection with pc3-GD3s by
RT-PCR (Fig. 4A). As shown by the relative band intensities with each $\beta$-actin control (Fig. 4B), the level of ICAM-1 expression was decreased. In addition, when the ER positive $(+)$ breast cancer cell line, MCF-7 cells (ER+) has been checked, the expression level of ICAM-1 protein was increased (Fig. 3C), indicating that the expression of GD3 and resultant GD2 conversion regulate the different expression of ICAM-1, depending on the ER negative or positive human breast cancer cells.

\section{Accumulation of the GD2 ganglioside in pc3-GD3s cells}

According to our previous study on MDA-MB231 cells, GD3/GD2 gangliosides belonging to the b-series were not expressed, while the a-series ganglioside GM3 was expressed at high levels [30]. Therefore, we compared the ganglioside composition of the pc3 and pc3-GD3s cells by HPTLC. As shown in Fig. 5A, the pc3-GD3s cells showed an accumulation of the b-series ganglioside GD2, but not GD3. In contrast, the levels of the a-series gangliosides GM3, GM2 and GM1 were reduced compared to the pc3 cells. The pc3 cells only expressed the a-series gangliosides GM3, GM2 and GM1, as expected. Also, 
the GM3 levels were decreased in pc3-GD3s cells compared to pc 3 cells ( $46.43 \%$ vs. $63.77 \%$ ). In contrast, GD2 levels showed a 32\% increase in GD3 synthase-transfected MDA-MB231 cells. These results were verified by measuring ganglioside composition using densitometry (Fig. 5B),

The GD2 ganglioside is synthesized from its precursor GD3 by N-acetylgalactosaminyltransferase ( $\beta 4$ GalNAc T; GM2/GD2 synthase). Therefore, we checked the mRNA level of GD2 synthase ( $\beta 4$ GalNAc T) by RT-PCR. Although the GD2 synthase mRNA was expressed in both of pc3-GD3s and pc3 cells, an increased expression level was observed in the pc3-GD3s cells (Fig. 5C). The mRNA of GT3 synthase (ST8SiaV), an enzyme required for c-series ganglioside production was not detected in either cell line (data not shown). These results suggested that the GD3 synthase-transfected cells accumulated GD2 ganglioside produced from GD3 precursors.

\section{Discussion}

Gangliosides on the outer leaflet of the plasma membrane of cells play important roles in biological processes such as 'tumor growth control', 'masking', 'tumor phenotype', 'receptor on-off' and 'cell-cell recognition' $[31,32,33]$. Various studies have shown that gangliosides and tumor invasion are deeply related $[34,35,36]$. For example, gangliosides such as GD3 and GD1b were reported to induce apoptosis in human breast tumor cells [35].

\section{(B)}

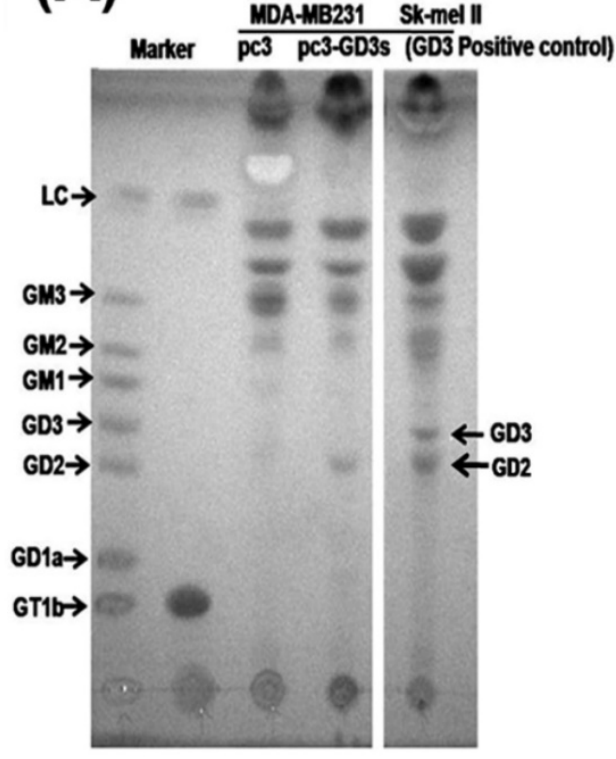

\section{(C)}
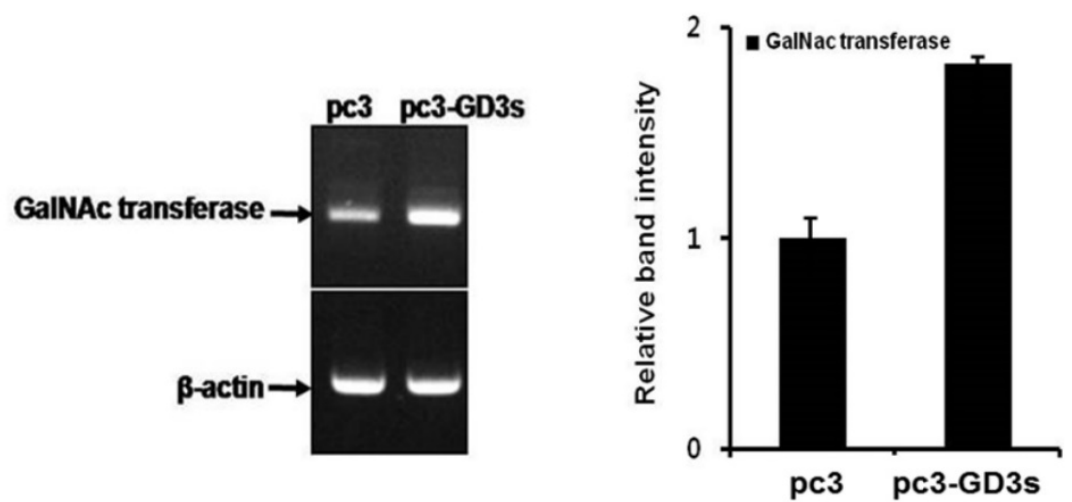

Figure 5. Accumulation of the GD2 ganglioside in pc3-GD3s cells. (A) The total ganglioside on a HPTLC plate. (B) Comparison of ganglioside contents in pc3 and pc3-GD3s cells. Percentage of each ganglioside was measured by densitometry with total gangliosides of each lane as $100 \%$. (C) Total mRNA was isolated from pc3 and pc3-GD3s cells. GD2 synthase ( $\beta 4-G a I N c$ T) was detected by RT-PCR (30 cycles). The bar graphs represent the relative band intensity obtained from the RT-PCR results by densitometry. 
The GD3 has been described as the Greek goddess Hecate [37], referring to the different functions of GD3 in cells. GD3 was reported to be a tumor-related antigen [38] and functions as a pro-apoptotic molecule [9]. GM3 and GD3 are highly expressed in the most common malignant breast cancer. The expression of GD3 synthase was also reported to be highly associated with breast cancer [12]. However, there are fundamental differences between human breast cancers and breast cancer cell line models such as MDA-MB231 in their glycosylation [30, 39]. For example, three different breast cell such as MCF-7, SK-BR3 and MDA-MB231 scarcely expressed GD3 synthase. These results led us to attempt to study the relationship between GD3 expression and breast cancer invasion.

In this study, we established GD3 synthase-overexpressing cells (pc3-GD3s) in MDA-MB231 cells (Fig. 1B, C, D) and examined cell behavior of pc3-GD3s cells under $1 \mathrm{mg} / \mathrm{ml}$ G418 bulk selection. The growth of pc3-GD3s cells was not different from the pc3 cells (Fig. 2A). Cell proliferation is not affected by the specific ICAM1-1 siRNA (data not shown), when MDA-MB231 cells were transfected with ICAM-1 siRNA. Similar results using MCF-7 breast cells were also observed, indicating that ICAM-1 is not related to the cell growth. Interestingly, the similar results are also reported in the recent report what used the MDA-MB-231 cells [20]. However, the morphology of the pc3-GD3s cells significantly changed when compared to the control (Fig. 2B). This result suggested that the overexpressed GD3 synthase can induce phenotypic changes in human breast cancer MDA-MB231 cells.

Invasion of breast cancer is important during the process of metastasis, since cell invasion and emigration activity determine the loss and gain of cell surface molecules [20,40]. In breast cancer invasion, ICAM-1 is associated with inflammatory and immune responses, as well as with epithelial tumorigenesis $[16,20]$ and metastatic potential of breast cancer cells [21]. Then, we investigated the anti-metastatic potential of GD3 synthase-overexpressing MDA-MB231 cells as the ER negative cells. In in vitro invasion assay, invasion activity was markedly suppressed in the pc3-GD3s cells (Fig. 2C). ICAM-1 expression was significantly down-regulated in pc3-GD3s cells (Fig. 3A, B, C, D, E), suggesting that over-expression of GD3 synthase suppresses invasion activity by down-regulation of ICAM-1. Interestingly, when SK-BR3 cells were used as another type of ER negative human breast cancer cell line, the SK-BR3 (ER-) cells exhibited the reduced level of ICAM-1 expression likely to MDA-MB231 cells, which are also ER negative breast cancer cells [2] (Fig. 4). In contrast, the ER positive (+) MCF-7 cells (ER+) [2] showed the increased expression level of ICAM-1. This clearly suggested that the estrogen receptor is indeed a co-regulator to regulate the ICAM-1 expression with GD3 to GD2 conversion in human breast cancer cells. Thus, it is suggested that GD2 may be a key regulator to downregulate the functional ICAM-1, intrinsically to cause the downregulated invasive potential in ER negative cell lines. As ICAM-1 has recently been suggested as a molecular target for triple negative breast cancer [41], the relationship between the ER and sialylated glycolipids is of interest for further elucidation in breast cancer biology. We have further tested various signaling pathways for potential influence on ICAM-1 expression, since ICAM-1 is reported to be regulated through several primary pathways: AKT, ERK and p38 activation [42, 43]. Phosphorylation of ERK and p38 was not changed in pc3-GD3s cells, but the phosphorylation of AKT was inhibited in the pc3-GD3s compared to the control (pc3) (Fig. 3F).

In our previous paper [30], the over-expression of GD3 synthase in MDA-MB231 cells was shown to lead to expression of $\mathrm{b}$ - and c-series gangliosides including GD3, GD2 and GT3 [30]. Here, our results showed the accumulation of the ganglioside GD2 instead of GD3 in GD3 synthase overexpressed cells (pc3-GD3s) (Fig. 5A). During the establishment of the stable pc3-GD3s transfectants, many different sialic acid-containing gangliosides were observed in the pc3-GD3s cells through the enforced GD3 synthase expression. Interestingly, our previous result [30] is a little bit opposite, as the GD3 synthase gene transfectants express and accumulate mainly GD3, GD2 and GT3 gangliosides when detected using FACS analysis in the initial stage. However, the absolute finding of the present study is that intracellular ganglioside GD3 is transformed to GD2 type, probably by intracellular endogenous GD2 synthase, more specifically Galactose- $\beta 1,4$-GalNAc transferase. This is a new finding of the present study and the GD2 synthase or Gal- $\beta 1,4-G a l N A c$ transferase-mediated GD2 synthesized from GD3 in the stably transfected pc3-GD3s cells triggers the inhibited expression of the ICAM-1. Therefore, it is interest to note that the GD3s transfectants further activate the downstream gangliosides synthesis, like GD2 in the stable pc3-GD3s cells, accumulating the GD2 in the breast cancer MDA-MB231 cells. This difference between the two different transfectant is caused by the present bulk selection or the previous one-colony selection. Therefore, composition of gangliosides could be affected by cell selective conditions. 


\section{Supplementary Material}

Supplementary figure 1.

http://www.ijbs.com/v13p0265s1.pdf

\section{Acknowledgments}

The present study was in part supported from the Basic Science Research Program through National Research Foundation of Korea (NRF) grant, funded by the Ministry of Education, Science and Technology (MEST) of Korea (NRF-2014R1A1A2007049 to TWC, NRF-2015R1D1A1A01057153 to CHK, NRF-2016R1D1A1B03932753 to TWC). The funders had no role in study design, data collection, and analysis, decision to publish, or preparation of the manuscript. All of authors declare that there are no conflicts of interest. TWC and CHK (Cheorl-Ho Kim) designed the research; $\mathrm{KMK}, \mathrm{KWK}$, TWC, $\mathrm{SHH}$ and CHK (Choong-Hwan Kwak) performed the research; TWC, KTH, SHC, MJL, YCL and CHK (Cheorl-Ho Kim) analyzed the data; KMK and CHK (Cheorl-Ho Kim) wrote the article.

\section{Competing Interests}

The authors have declared that no competing interest exists.

\section{References}

[1] Lloyd KO, Furukawa K. Biosynthesis and functions of gangliosides: recent advances. Glycoconj J. 1998; 15: 627-36.

[2] Ha SH, Lee JM, Kwon KM, Kwak CH, Abekura F, Park JY, et al. Exogenous and Endogeneous Disialosyl Ganglioside GD1b Induces Apoptosis of MCF-7 Human Breast Cancer Cells. Int J Mol Sci. 2016; 17(5): pii: E652.

[3] Moon SK, Kim HM, Lee YC, Kim CH. Disialoganglioside (GD3) synthase gene expression suppresses vascular smooth muscle cell responses via the inhibition of ERK1/2 phosphorylation, cell cycle progression, and matrix metalloproteinase-9 expression. J Biol Chem. 2004; 279: 33063-70.

[4] Ha KT, Lee YC, Kim CH. Overexpression of GD3 synthase induces apoptosis of vascular endothelial ECV304 cells through downregulation of Bcl-2. FEBS Lett. 2004; 568: 183-7.

[5] Moon SK, Kang SK, Kim CH. Reactive oxygen species mediates disialoganglioside GD3-induced inhibition of ERK1/2 and matrix metalloproteinase-9 expression in vascular smooth muscle cells. FASEB J. 2006; 20: 1387-95.

[6] Yu RK, Macala LJ, Taki T, et al. Developmental changes in ganglioside composition and synthesis in embryonic rat brain. J. Neurochem 1988; 50: 1825-9.

[7] Kang NY, Kim CH, Kim KS, Ko JH, Lee JH, Jeong YK, et al. Expression of the human CMP-NeuAc:GM3 alpha2,8-sialyltransferase (GD3 synthase) gene through the NF-kappaB activation in human melanoma SK-MEL-2 cells. Biochim Biophys Acta 2007; 1769: 622-30.

[8] Kwon HY, Dae HM, Song NR, Kim KS, Kim CH, Lee YC. Valproic acid induces transcriptional activation of human GD3 synthase (hST8Sia I) in SK-N-BE(2)-C human neuroblastoma cells. Mol Cells 2009; 27: 113-8.

[9] Maria RD, Lenti L, Malisan F, d'Agostino F, Tomassini B, Zeuner A, et al Requirement for GD3 ganglioside in CD95- and ceramide-induced apoptosis. Science 1997; 277: 1652-5

[10] Giammarioli AM, Garofalo T, Sorice M, Misasi R, Gambardella L, Gradini R, et al. GD3 glycosphingolipid contributes to Fas-mediated apoptosis via association with ezrin cytoskeletal protein. FEBS Lett. 2001; 506: 45-50.

[11] Marquina G, Waki H, Fernandez LE, Kon K, Carr A, Valiente O, et al. Gangliosides expressed in human breast cancer. Cancer Res. 1996; 56: 5165-71.

[12] Ruckhaberle E, Rody A, Engels K, Gaetje R, Minckwitz GV, Schiffmann S, et al. Microarray analysis of altered sphingolipid metabolism reveals prognostic significance of sphingosine kinase 1 in breast cancer. Breast Cancer Res Treat. 2008; 112: 41-52.

[13] Cazet A, Bobowski M, Rombouts Y, Lefebvre J, Steenackers A, Popa I, et al. The ganglioside $\mathrm{G}(\mathrm{D} 2)$ induces the constitutive activation of c-Met in MDA-MB-231 breast cancer cells expressing the G(D3) synthase. Glycobiology $2012 \cdot 22 \cdot 806-16$
[14] Sarkar TR, Battula VL, Werden SJ, Vijay GV, Ramirez-Peña EQ, Taube JH, et al. GD3 synthase regulates epithelial-mesenchymal transition and metastasis in breast cancer. Oncogene 2015; 34: 2958-67.

[15] Battula VL, Shi Y, Evans KW, Wang RY, Spaeth EL, Jacamo RO, et al. Ganglioside GD2 identifies breast cancer stem cells and promotes tumorigenesis. J Clin Invest. 2012; 122: 2066-78.

[16] Martinez C, Hofmann TJ, Marino R, Dominici M, Horwitz EM. Human bone marrow mesenchymal stromal cells express the neural ganglioside GD2: a novel surface marker for the identification of MSCs. Blood 2007; 109: $4245-4248$

[17] Hubbard AK, Rothlein R. Intercellular adhesion molecule-1 (ICAM-1) expression and cell signaling cascades. Free Radic Biol Med. 2000; 28: 1379-86.

[18] Pantel K, Schlimok G, Angstwurm M, Passlick B, Izbicki JR, Johnson JP, et al. Early metastasis of human solid tumours: expression of cell adhesion molecules. Ciba Found Symp. 1995; 189: 157-70

[19] O'Hanlon DM, Fitzsimons H, Lynch J, Tormey S, Malone C, Given HF. Soluble adhesion molecules (E-selectin, ICAM-1 and VCAM-1) in breast carcinoma. Eur J Cancer 2002; 38: 2252-7.

[20] Rosette C, Roth RB, Oeth P, Braun A, Kammerer S, Ekblom J, et al. Role of ICAM1 in invasion of human breast cancer cells. Carcinogenesis 2005; 26: 943-50.

[21] Elangbam CS, Qualls CW. Dahlgren, R.R. Cell adhesion molecules-update. Vet Pathol. 1997; 34: 61-73.

[22] Schröder C, Witzel I, Müller V, Krenkel S, Wirtz RM, Jänicke F. Prognostic value of intercellular adhesion molecule (ICAM)-1 expression in breast cancer. J Cancer Res Clin Oncol. 2011; 137: 1193-1201.

[23] Weigelt B, Peterse JL, Veer LJV. Breast cancer metastasis: markers and models. Nat Rev Cancer 2005; 5: 591-602.

[24] Nizamutdinova IT, Lee GW, Lee JS, Cho MK, Son KH, Jeon SJ, et al. Tanshinone I suppresses growth and invasion of human breast cancer cells, MDA-MB-231, through regulation of adhesion molecules. Carcinogenesis 2008; 29: 1885-92.

[25] Chung TW, Lee YC, Ko JH, Kim CH. Hepatitis B Virus X protein modulates the expression of PTEN by inhibiting the function of p53, a transcriptional activator in liver cells. Cancer Res. 2003; 63: 3453-8.

[26] Zeng G, Li DD, Gao L, Birkle S, Bieberich E, Tokuda A, et al. Alteration of ganglioside composition by stable transfection with antisense vectors against GD3-synthase gene expression. Biochemistry 1999; 38: 8762-9.

[27] Nakamura K, Suzuki M, Taya C, Inagaki F, Yamakawa T, Suzuki A. A sialidase-susceptible ganglioside, IV3 alpha(NeuGc alpha 2-8NeuGc)-Gg4Cer, is a major disialoganglioside in WHT/Ht mouse thymoma and thymocytes. J Biochem. 1991; 110: 832-41.

[28] Scambia G, Ranelletti FO, Panici PB, Piantelli M, De Vincenzo R, Ferrandina G, et al. Quercetin induces type-II estrogen-binding sites in estrogen-receptor-negative (MDA-MB231) and estrogen-receptor-positive (MCF-7) human breast-cancer cell lines. Int J Cancer 1993; 54: 462-6.

[29] Min JK, Lee YM, Kim JH, Kim YM, Kim SW, Lee SY, et al. Hepatocyte growth factor suppresses vascular endothelial growth factor-induced expression of endothelial ICAM-1 and VCAM-1 by inhibiting the nuclear factor-kappaB pathway. Circ Res. 2005; 96: 300-7.

[30] Cazet A, Groux-Degroote S, Teylaert B, Kwon KM, Lehoux S, Slomianny C. et al. GD3synthase overexpression enhances proliferation and migration of MDA-MB-231 breast cancer cells. Biol Chem. 2009; 390: 601-9.

[31] Varki A. Biological roles of oligosaccharides: all of the theories are correct. Glycobiology 1993; 3: 97-130.

[32] Svennerholm L. Gangliosides and synaptic transmission. Adv Exp Med Biol. 1980; 125: 533-44.

[33] Hakomori S, Igarashi Y. Gangliosides and glycosphingolipids as modulators of cell growth, adhesion, and transmembrane signaling. Adv Lipid Res. 1993; 25: 147-62.

[34] Potapenko M, Shurin GV, de Leon J. Gangliosides as immunomodulators. Adv Exp Med Biol. 2007; 601: 195-203.

[35] Ma R, Koulov A, Moulton C, Basu M, Banerjee S, Goodson H, et al. Apoptosis of human breast carcinoma cells in the presence of disialosyl gangliosides: II. Treatment of SKBR3 cells with GD3 and GD1b gangliosides. Glycoconj J. 2004; 20: 319-30.

[36] Hoon DS, Banez M, Okun E, Morton DL, Irie RF. Modulation of human melanoma cells by interleukin- 4 and in combination with gamma-interferon or alpha-tumor necrosis factor. Cancer Res. 1991; 51: 2002-8.

[37] Malisan F, Testi R. The ganglioside GD3 as the Greek goddess Hecate: several faces turned towards as many directions. IUBMB Life 2005; 57: 477-82.

[38] Portoukalian J, Zwingelstein G, Dore JF. Lipid composition of human malignant melanoma tumors at various levels of malignant growth. Eur J Biochem. 1979; 94: 19-23.

[39] Julien S, Krzewinski-Recchi MA, Harduin-Lepers A, Gouyer V, Huet G, Le Bourhis $X$, et al. Expression of sialyl-Tn antigen in breast cancer cells transfected with the human CMP-Neu5Ac: GalNAc alpha2,6-sialyltransferase (ST6GalNac I) cDNA. Glycoconj J. 2001; 18: 883-93.

[40] Weigelt B, Glas AM, Wessels LF, Witteveen AT, Peterse JL, Veer LJV. Gene expression profiles of primary breast tumors maintained in distant metastases. Proc Natl Acad Sci USA 2003; 100: 15901-5.

[41] Guo P, Huang J, Wang L, Jia D, Yang J, Dillon DA, et al. ICAM-1 as a molecular target for triple negative breast cancer. Proc Natl Acad Sci USA 2014; 111: 14710-14715. 
[42] Minhajuddin M, Bijli KM, Fazal F, Sassano A, Nakayama KI, Hay N, et al. Protein kinase C-delta and phosphatidylinositol 3-kinase/Akt activate mammalian target of rapamycin to modulate NF-kappaB activation and intercellular adhesion molecule-1 (ICAM-1) expression in endothelial cells. J Biol Chem. 2009; 284: 4052-61.

[43] Calixto JB, Campos MM, Otuki MF, Santos AR. Anti-inflammatory compounds of plant origin. Part II. Modulation of pro-inflammatory cytokines, chemokines and adhesion molecules. Planta Med. 2004; 70: 93-103.

[44] Arcari PA, Martinelli R, Salvatore F. The complete sequence of a full length cDNA for human liver glyceraldehyde-3-phosphate dehydrogenase: evidence for multiple mRNA species. Nucleic Acids Res. 1984; 12: 9179-89. 